\title{
Dual inoculation with mycorrhizal and saprotrophic fungi suppress the maize growth and development under phenanthrene exposure
}

Claudio Lagos ${ }^{1,2}$, John Larsen ${ }^{3}$, Estefanía Saucedo Correa ${ }^{3}$, Leonardo Almonacid ${ }^{1}$, Hector Herrera $^{1}$, Alejandra Fuentes ${ }^{1}$, Cesar Arriagada ${ }^{1 *}$

${ }^{1}$ Laboratorio de Biorremediación, Facultad de Ciencias Agropecuarias y Forestales, Universidad de La Frontera, Francisco Salazar 01145, Temuco, Chile. ${ }^{2}$ Programa de Doctorado en Ciencias de Recursos Naturales, Universidad de la Frontera, Casilla 54-D, Temuco, Chile. Francisco Salazar 01145, Temuco, Chile. ${ }^{3}$ Laboratorio de Agroecología, Instituto de Investigaciones en Ecosistemas y Sustentabilidad, Universidad Nacional Autónoma de México, Morelia. Antigua Carretera a Pátzcuaro 8701, 58190, Morelia, Michoacán, México. *Corresponding author: cesararriagada@ufrontera.cl

\begin{abstract}
Polycyclic aromatic hydrocarbons (PAHs) belong to the group of persistent organic pollutants with detrimental impact on the environment and its living organisms. Soil acts as the main PAH sink why strategies to remediate PAH contaminated soil are needed. Inoculation with plant growth promoting and stress alleviating fungi such as arbuscular mycorrhizal (AM) fungi and saprotrophic fungi could be an efficient bioremediation strategy for PAH contaminated soils, but information about their compatibility is missing. In a greenhouse pot experiment we examined interactions between the AM fungus Rhizophagus irregularis and the saprotrophic fungus Trichoderma viride and their single and dual effects on maize plant growth performance and communities of rhizosphere microorganisms in a Phenanthrene spiked soil. Phenanthrene reduced maize plant growth independent of both fungal inoculants, which were also both inhibited by Phenanthrene. On the other hand strong mutual inhibition between $R$. irregularis and T.viride was observed, which mitigated their plant growth promoting effects independent of Phenanthrene. In conclusion, the observed mutual inhibition between $R$. irregularis and $T$. viride underline the importance of testing for compatibility between microbial inoculants when these are used in combination.
\end{abstract}

Keywords: Arbuscular mycorrhiza, Fungal interactions, Polycyclic aromatic hydrocarbons, Rhizozphere, Trichoderma. 


\section{Introduction}

Polycyclic aromatic hydrocarbons (PAHs) are organic pollutants widely distributed in different ecosystems, highly persistent and toxic for living organisms because of their carcinogenic, mutagenic and teratogenic properties (Maliszewska-Kordybach, 1999). Soil is the main sink of PAHs hosting almost $90 \%$ of PAHs produced, which are deposited into the soil from the atmosphere, and once in the soil environment, PAHs are strongly sorbed depending on the content of organic matter in soil. Also, PAHs can be dissipated from the soil by photo-oxidation, bioaccumulation in plant tissues, leaching, biosorption and biodegradation by soil microorganisms (Vácha et al., 2010).

Plants play a key role in soil PAH dissipation via their enhancement on PAH degrading rhizosphere microorganisms leading to an increase in soil enzymatic activity such as polyphenol oxidase, dehydrogenase, urease, peroxidase and tyrotinase that are involved in the degradation of PAH (Liu et al., 2015). Also bioaccumulation of PAH in plant tissue contribute to their dissipation from soil. Nevertheless, successful phytoremediation depends on how the plants and their root associated microorganisms respond to the pollutant (Parrish et al, 2006).

Among these root associated microorganisms, arbuscular mycorrhizal (AM) fungi form biotrophic symbiosis with roots, which is characterized by a reciprocal exchange of nutrients, where the fungus receive carbohydrates from the host and the AM fungus provide the host with mineral nutrients mainly $\mathrm{P}$ via the extraradical mycelium. Besides improving host nutrition AM fungi are also known to alleviate the plant host from biotic and abiotic stress (Smith and Read, 2008; Cornejo et al., 2017). Plant stress alleviation by AM fungi has been shown for many soil contaminants, including heavy metals and persistent organic pollutants
(Fuentes et al., 2016). AM fungi have been shown to increase soil PAH dissipation both by promoting the soil microbial population leading to a PAH biodegradation and via accumulation of PAH in fungal tissue in roots (Gao et al., 2011).

On the other hand, saprotrophic fungi are key drivers of soil organic matter decomposition due to their ample enzymatic machinery also allowing degradation of recalcitrant organic compounds including soil pollutants such as PAHs (Mineki et al., 2015). Several species such as Trichoderma are widely known as plant health and growth promoters mainly linked to their production of biological active secondary metabolites including phytohormones and antibiotics against plant pathogens (Contreras-Cornejo et al., 2016).

Combination of AM and saprotrophic fungi such as Trichoderma spp has been reported to increase plant growth and yield (Yadav et al., 2015). Regarding soil pollution combination of AM and saprotrophic fungi has been tested mainly for heavy metals and metalloids (Arriagada et al., 2009; Fuentes et al., 2016), while information on combined inoculation with AM and saprotrophic fungi to mitigate the adverse impacts of PAHs on plant growth and possible bioremediation is limited.

The objective of this study was to examine interactions between the AM fungus Rhizophagus irregularis and the saprotrophic fungus Trichoderma viride and their single and combined effects on maize plant growth and soil microbial communities in soil with and without the PAH Phenanthrene. The main hypothesis was that dual inoculation with $R$. irregularis and T. viride would improve plant growth and mitigate plant stress from Phenanthrene application to a higher extent than from single fungal inoculation. 


\section{Material and Methods}

\subsection{Experimental design}

A completely randomized factorial greenhouse pot experiment was performed with the three factors: 1) Arbuscular mycorrhizal (AM) fungus (with or without Rhizophagus irregularis); 2) Saprotrophic fungus (with or without Trichoderma viride) and 3) Phenanthrene $\left(0\right.$ and $\left.250 \mathrm{mg} \mathrm{kg}^{-1}\right)$. Each of the eight treatments had five replicates resulting in a total of 40 experimental units.

\subsection{Biological materials}

Soil was obtained from the experimental field station of the National Agricultural University of Mexico, Campus Morelia, Michoacán, Mexico. Soil texture was clayish (53.2\% clay, $27.3 \%$ silt and $19.5 \%$ sand) and with the following chemical characteristics: $2.7 \%$ organic matter, $23.2 \mathrm{mg} \mathrm{kg}^{-1}$ inorganic nitrogen, 5.8 mg kg-1 available phosphorus (Olsen $\mathrm{P})$ and $\mathrm{pH}\left(\mathrm{H}_{2} \mathrm{O}\right)$ 7.3. Soil was mixed with quartz sand (1:1,w:w) and disinfected in an autoclave in two consecutive days. Mineral fertilization except $\mathrm{P}$ was applied to the soil so that $\mathrm{P}$ was the only nutrient limiting plant growth (mg kg ${ }^{-1}$ dry soil): $\mathrm{K}_{2} \mathrm{SO}_{4}(370.3), \mathrm{CaCl}_{2} \times 2 \mathrm{H}_{2} \mathrm{O}(75.0)$, $\mathrm{CuSO}_{4} \times 5 \mathrm{H}_{2} \mathrm{O}(2.1), \mathrm{ZnSO}_{4} \times 7 \mathrm{H}_{2} \mathrm{O}(5.4), \mathrm{MnSO}_{4}$ x $\mathrm{H}_{2} \mathrm{O}$ (10.5), $\mathrm{MgSO}_{4}$ x $7 \mathrm{H}_{2} \mathrm{O}$ (405.4), $\mathrm{Na}_{2} \mathrm{MoO}_{4}$ x $2 \mathrm{H}_{2} \mathrm{O}(0.18), \mathrm{NH}_{4} \mathrm{NO}_{3}(285.71)$.

The Zea mays L. hybrid DK2061 was used in the present study. In each pot three seeds were sown, but after seedling emergence, one week after sowing, pots were unified so that only one seedling was left in each pot. The AM Rhizophagus irregularis (BEG87) and the saprotrophic fungus Trichoderma viride (BAFC8850) were obtained from the culture collection at the Agroecology Laboratory at the Universidad Nacional Autonoma de México, Campus Morelia, México. Inocu- lum of $R$. irregularis was obtained from a maize trap culture consisting of soil with root segments, mycelium and spores.

Inoculum of $T$. viride consisted of conidia obtained from two week old cultures with potato dextrose agar. Conidia were suspended in Milli-Q water and the counted by microscopy using a hemocytometer.

\subsection{Experimental setup}

The experiment was carried out using the bi-compartmented system, hyphal compartments (HCs) and root + hyphal compartments (RHC). Pots were filled with $1 \mathrm{~kg}$ of soil:sand mix and AM fungal inoculum $(10 \%, \mathrm{w} / \mathrm{w})$ was mixed into the soil in the treatments with $R$. irregularis. Then two empty tubes $(200 \mathrm{ml})$ were inserted in the soil in each pot oppositely to the center, where the seeds were sown. Two weeks after seedling emergence plants were thinned to one seedling per pot. Three weeks after sowing, when the mycorrhizal association was supposed to be established, tubes were removed and replaced by mesh bags made from nylon mesh of the same shape. One mesh bag with $20 \mu \mathrm{m}$ mesh diameter, allowing entrance of hyphae but not roots (HCs) and another mesh bag with $400 \mu \mathrm{m}$ mesh diameter allowing entrance of roots and hyphae (RHC). Each set of mesh bags were filled with $100 \mathrm{~g}$ soil:sand substrate spiked with Phenanthrene (250 mg kg-1 soil) in the corresponding Phenanthrene treatments. Phenanthrene dissolved in acetone $(25 \mathrm{mg}$ Phenanthrene in $5 \mathrm{ml}$ acetone) was thoroughly mixed in to the soil (100 g) and left to evaporate for 48 hours in a fume hood. The same amount of acetone was applied to the soil in the treatments without Phenanthrene. Hereafter inoculum of $T$. viride ( $1 \times 10^{6}$ conidian $\mathrm{g}^{-1}$ soil) was also mixed into the soil:sand substrate. Finally, the filled mesh bags were inserted into pots according to the experimental design. 


\subsection{Plant growth conditions}

Plants were grown under greenhouse conditions with minimum and maximum temperature of $15^{\circ} \mathrm{C}$ and 30 ${ }^{\circ} \mathrm{C}$, respectively. Watering was made daily by weight to $70 \%$ of the water holding capacity throughout the experiment. Plants were harvested 10 weeks after sowing. Mesh bags were carefully removed from the pots and the soil within the mesh bags was collected and stored according to the respective measurements. Roots were gently washed free of growth substrate and separated from the shoot. Dry weights of shoots and roots were obtained after drying at $80{ }^{\circ} \mathrm{C}$ for 48 hr. Prior drying of roots, they were cut into 5-to 10 $\mathrm{mm}$ segments and a representative two-gram root subsample was taken for measurement of AM root colonization.

\subsection{Population density of $T$. viride}

From both mesh bags and the pot one gram of soil was individually applied to a $15 \mathrm{ml}$ Falcon tube with $9 \mathrm{ml}$ sterile Milli-Q water and Triton (0.01\%) and here after serial dilutions were made up to $10^{-3}$ from which $0.1 \mathrm{ml}$ was placed on Trichoderma selective medium according to Elad et al. (1981, with modifications). Plates were incubated in darkness at $27{ }^{\circ} \mathrm{C}$ and observed on a daily basis. Population density of $T$. viride was calculated according to the amount of soil in the respective dilutions and expressed as $\mathrm{CFU} \mathrm{g} \mathrm{g}^{-1}$ dry soil.

\subsection{Arbuscular mycorrhizal root colonization}

Two gram of fresh roots from all experimental units was stained with trypan blue according to Phillips and Hayman (1970) and AM root colonization was measured by microscopy according to Giovanetti and Mosse (1980).

\subsection{Biomarker fatty acids}

Ten gram of soil samples were freeze dried and powderized in a mortar of which one-gram subsamples were used for extracting of whole cell fatty acids according to the method of Sasser (1990). To enable quantification of the extracted fatty acid methyl esters, a known amount of an internal standard, nonadecanoate fatty acid methyl ester 19:0 was added to each sample. Analyses of fatty acid methyl esters were performed using the software package Sherlock Version 6.0 (MIDI Inc.). Hydroxylic and cyclic fatty acids were used as biomarkers for Gram-negative bacteria, fatty acids with branched chains localized on positions iso, and anteiso were used as Gram-positive bacteria biomarkers and methylated fatty acids were used a biomarker for Actinobacteria (Ratledge and Wilkinson, 1988). The fatty acid 16:105 and 18:2 $\omega 6,9$ were used as biomarkers for AM (Olsson et al., 1999) and saprotrophic fungi (Frostegård and Bååth, 1996), respectively. Fatty acid analysis was performed with the Agilent gas chromatograph 7890 B.

\subsection{Statistical analyses}

Data were analyzed according to the factorial design with three way analyses of variance. Statistical significance was determined at $\mathrm{P}<0.05$. Data sets were tested for normality and variance homogeneity with Kolmogorov Smirnov and Cochran's C tests respectively and log transformation was applied when necessary. The variable AM root colonization was arcsine transformed before statistical analysis. All statistical analyses were performed with the SPSS software, version 11.0 (SPSS Inc., 1989-2001) (Visauta, 1998). 


\section{Results}

\subsection{Shoot and root dry weight}

Significant "AM x saprotrophic fungus" interactions and single factor Phenanthrene effects were obtained for both shoot and root dry weight (Table 1). Application of Phenanthrene reduced shoot and root dry weigt independent of fungal inoculation. Highest shoot dry weight was found in plants inoculated with $R$. irregularis, which was however reduced after dual inoculation with $T$. viride, independent of Phenanthrene application Individual inoculation with $R$. irregularis and $T$. viride caused improved root dry weight, which was mitigated by dual fungal inoculation, again independent of Phenanthrene application.

Table 1. Significance of the main treatments on plant biomass based on factorial ANOVA.

\begin{tabular}{|c|c|c|c|c|c|c|c|}
\hline & R.i & T.v & PHE & R.i x T.v & R.i $\times$ PHE & T.v $\times$ PHE & R.i $\times$ T.v $\times$ PHE \\
\hline \multicolumn{8}{|l|}{$F$-values } \\
\hline Shoot dry weight & $0.49 \mathrm{n} . \mathrm{s}$ & $5.72^{*}$ & $5.95^{*}$ & $4.85^{*}$ & $0.57 \mathrm{n} . \mathrm{s}$ & $0.28 \mathrm{n} . \mathrm{s}$ & $0.2 \mathrm{n} . \mathrm{s}$ \\
\hline Root dry weight & $0.24 \mathrm{n} . \mathrm{s}$ & $6.2^{*}$ & $32.62 * * *$ & $51.6 * * *$ & $3.31 \mathrm{n.s}$ & $3.35 \mathrm{n} . \mathrm{s}$ & $0.96 \mathrm{n} . \mathrm{s}$ \\
\hline
\end{tabular}

R.i: R. irregularis; T.v: T. viride; PHE: Phenanthrene. n.s: Not significant. ${ }^{*} \mathrm{P}<0.05 ; * * \mathrm{P}<0.01,{ }^{*} * * \mathrm{P}<0.001$

\subsection{Population density of $T$. viride}

In the RHC a significant "AM x Phenanthrene" interaction was obtained for the population density of $T$. viride (Table 2). Inoculation with $R$. irregu- laris reduced the population density of $T$. viride, which was further suppressed in combination with Phenanthrene application, though Phenanthrene alone had no effect on $T$. viride population density (Figure 1).

Table 2. Significance of the main treatments on Trichoderma CFU and AM colonization based on factorial ANOVA.

\begin{tabular}{lccc}
\multicolumn{4}{c}{ Trichoderma CFU } \\
\hline & R.i & PHE & R.i $\times$ PHE \\
\hline F-values & & & \\
NCS & $15.92^{* * *}$ & $9.42^{* *}$ & $0.28 \mathrm{n} . \mathrm{s}$ \\
RHC & $76.54^{* * *}$ & $26.04^{* * *}$ & $7.30^{*}$ \\
HC & $2.45 \mathrm{n} . \mathrm{s}$ & $39.72^{* * *}$ & $3.61 \mathrm{n} . \mathrm{s}$ \\
& & & \\
\hline
\end{tabular}

\begin{tabular}{lccc}
\multicolumn{4}{c}{ AM colonization } \\
\hline & T.v & PHE & T.v x PHE \\
\hline F-values & & & \\
NCS & $13.2^{* *}$ & $50.61^{* * *}$ & 0.10 n.s \\
RHC & 2.47 n.s & $43.11^{* * *}$ & $25.07^{* * *}$ \\
HC & - & - & - \\
& & & \\
\hline
\end{tabular}

R.i: R. irregularis; PHE: Phenanthrene; T.v: T. viride. NCS: Not compartmented soil; RHC: Root and hyphal compartment; HC: Hyphal compartment. n.s: Not significant. ${ }^{*} \mathrm{P}<0.05 ; * * \mathrm{P}<0.01, * * * \mathrm{P}<0.001$ 
In the HC significant single factor effects where observed for both factors AM fungi and Phenanthrene, which also both reduced the population density of $T$. viride (Figure 1). In the NCS, where T. viride was not inoculated a significant reduction from Phenanthrene application was found independent of inoculation with $R$. irregularis (Table 1, Figure 1).
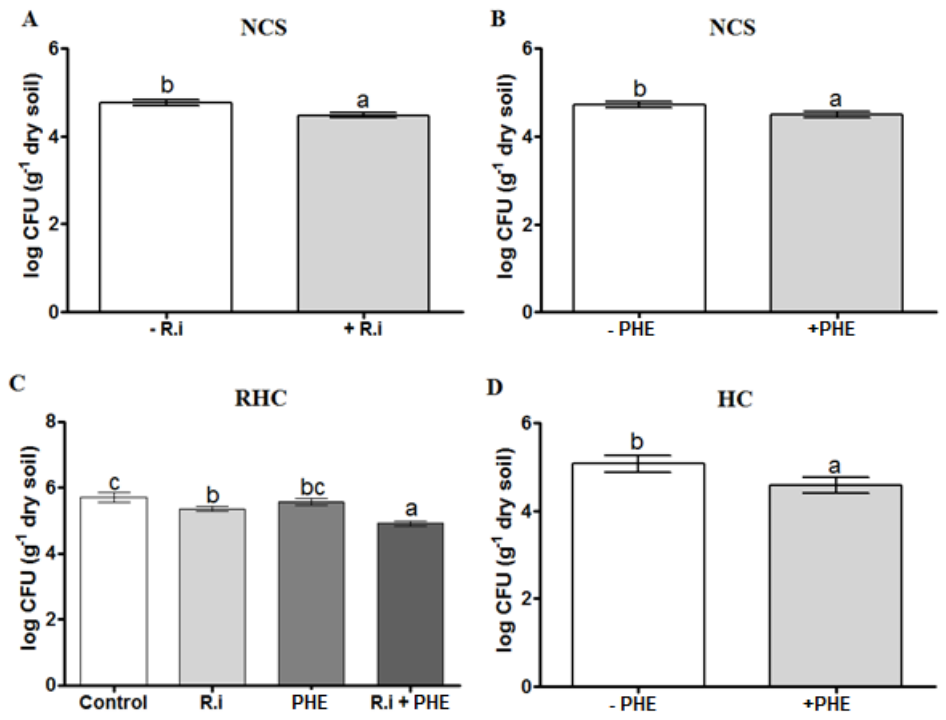

Figure 1. Factor treatments means for population of T. viride (CFU g-1 dry soil) in the different compartments. A) Effect of $R$. irregularis in NCS. B) Effect of phenanthrene in NCS. C) Effect of factor interaction comprised by R. irregularis and phenanthrene in RHC. D) Effect of phenanthrene in HC. NCS: Non-compartmented soil; RHC: Root and hyphal compartments; HC: Hyphal compartments; R.i: R. irregularis; PHE: Phenanthrene. Different letters indicate significant factor effects and error bars represent standard error of the mean.

\subsection{AM root colonization}

A significant "Saprotrophic fungus x Phenanthrene" was observed for $\mathrm{AM}$ root colonization in roots from the RHC (Table 2). Single inoculation with $T$. viride and individual application of Phenanthrene had no effect on AM root colonization, whereas combination of Phenanthrene and $T$. viride reduced AM root colonization (Figure 2). In the NCS single factor effects where observed for both factors saprotrophic fungus and Phenanthrene, which both reduced the AM root colonization (Figure 2). 

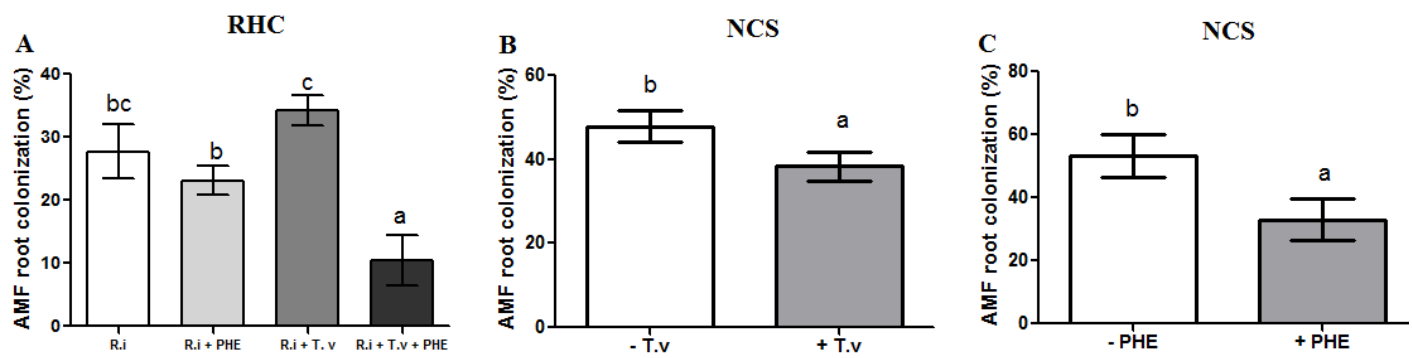

Figure 2. Factor treatments means for AMF root colonization in the different compartments. A) Effect of factor interaction comprised by phenanthrene and $T$. viride on AMF root colonization in RHC B) Effect of $T$. viride in NCS. C) Effect of phenanthrene in NCS. NCS: Non-compartmented soil; RHC: Root and hyphal compartments; R.i: R. irregularis; PHE: Phenanthrene; T.v: T. viride. Different letters indicate significant factor effects and error bars represent standard error of the mean.

\subsection{Biomarker fatty acids}

In the RHC significant saprotrophic fungus single factor effects were observed for Gram negative bacteria, Gram positive bacteria and actinobacteria, independent of the two other factors AM fungus and Phenanthrene (Table 3), whereas in the HCs the abundance of bacteria was unaffected by all three factors examined (Table 3). The abundance of Gram positive bacteria, Gram negative bacteria and actinobacteria in the RHC was higher in the factor treatment mean with $T$. viride compared to the factor treatment mean without $T$. viride (Figure 3).
A significant single factor effect of Phenanthrene was observed for the abundance of saprotrophic fungi in the HCs (Table 3), where application of Phenanthrene decreased the abundance of saprotrophic fungi (Figure 3). For the treatments with AM inoculation significant single factor effects with saprotrophic fungus and Phenanthrene were observed for the abundance of AM fungus in both RHC and HCs (Table 4). In both RHC and HCs inoculation with $T$. viride and application of Phenanthrene reduced the abundance of AM fungus (Figure 4). The background values of the AM biomarker fatty acid 16:1 $\omega 5$ in the RHC and the HCs were 1.07 and $0.73 \mathrm{~mol} \mathrm{~g}^{-1}$ dry soil, respectively. 
Table 3. Significance of the main treatments on whole cell fatty acids of different microorganisms in compartmented soil. A) Root and hyphal compartment. B) Hyphal compartment.

A

\begin{tabular}{lccccccc}
\hline & R.i & T.v & PHE & R.ix T.v & R.ix PHE & T.v x PHE & R.ix T.v x PHE \\
\hline F-values & & & & & & & \\
AMF & $20.43^{* * *}$ & $5.85^{*}$ & $11.31^{* *}$ & $6.16^{*}$ & $11.17^{* *}$ & $2.61 \mathrm{n} . \mathrm{s}$ & $3.27 \mathrm{n} . \mathrm{s}$ \\
SF & $0.05 \mathrm{n} . \mathrm{s}$ & $0.37 \mathrm{n} . \mathrm{s}$ & $1.54 \mathrm{n} . \mathrm{s}$ & $0.86 \mathrm{n} . \mathrm{s}$ & $0.43 \mathrm{n} . \mathrm{s}$ & $0.13 \mathrm{n} . \mathrm{s}$ & $0.02 \mathrm{n} . \mathrm{s}$ \\
Gram negative & $0.07 \mathrm{n} . \mathrm{s}$ & $6.80^{*}$ & $0.02 \mathrm{n} . \mathrm{s}$ & $1.13 \mathrm{n} . \mathrm{s}$ & $0.12 \mathrm{n} . \mathrm{s}$ & $0.39 \mathrm{n} . \mathrm{s}$ & $3.22 \mathrm{n} . \mathrm{s}$ \\
Gram possitive & $0.01 \mathrm{n} . \mathrm{s}$ & $12.88^{* *}$ & $1.23 \mathrm{n} . \mathrm{s}$ & $1.17 \mathrm{n} . \mathrm{s}$ & $0.05 \mathrm{n} . \mathrm{s}$ & $0.68 \mathrm{n} . \mathrm{s}$ & $3.89 \mathrm{n} . \mathrm{s}$ \\
Actinomycetes & $0.05 \mathrm{n} . \mathrm{s}$ & $11.08^{* *}$ & $0.27 \mathrm{n} . \mathrm{s}$ & $2.69 \mathrm{n} . \mathrm{s}$ & $2.16 \mathrm{n} . \mathrm{s}$ & $1.87 \mathrm{n} . \mathrm{s}$ & $2.33 \mathrm{n} . \mathrm{s}$ \\
\end{tabular}

B

\begin{tabular}{lccccccc}
\hline & & & & & & \\
& R.i & T.v & PHE & R.ix T.v & R.ix PHE & T.v x PHE R.ix T.v x PHE \\
\hline $\begin{array}{l}\text { F-values } \\
\text { AMF }\end{array}$ & $20.94^{* * *}$ & $2.93 \mathrm{n} . \mathrm{s}$ & $5.08^{*}$ & $3.20 \mathrm{n} . \mathrm{s}$ & $5.28^{*}$ & $0.46 \mathrm{n} . \mathrm{s}$ & $0.49 \mathrm{n} . \mathrm{s}$ \\
SF & $0.71 \mathrm{n} . \mathrm{s}$ & $0.55 \mathrm{n} . \mathrm{s}$ & $5.26^{*}$ & $1.52 \mathrm{n} . \mathrm{s}$ & $1.00 \mathrm{n} . \mathrm{s}$ & $1.91 \mathrm{n} . \mathrm{s}$ & $1.60 \mathrm{n} . \mathrm{s}$ \\
Gram negative & $0.00 \mathrm{n} . \mathrm{s}$ & $0.00 \mathrm{n} . \mathrm{s}$ & $0.16 \mathrm{n} . \mathrm{s}$ & $1.94 \mathrm{n} . \mathrm{s}$ & $0.00 \mathrm{n} . \mathrm{s}$ & $0.76 \mathrm{n} . \mathrm{s}$ & $0.02 \mathrm{n} . \mathrm{s}$ \\
Gram possitive & $0.54 \mathrm{n} . \mathrm{s}$ & $0.27 \mathrm{n} . \mathrm{s}$ & $0.02 \mathrm{n} . \mathrm{s}$ & $2.49 \mathrm{n} . \mathrm{s}$ & $0.62 \mathrm{n} . \mathrm{s}$ & $2.65 \mathrm{n} . \mathrm{s}$ & $0.99 \mathrm{n} . \mathrm{s}$ \\
Actinomycetes & $0.36 \mathrm{n} . \mathrm{s}$ & $0.52 \mathrm{n} . \mathrm{s}$ & $0.16 \mathrm{n} . \mathrm{s}$ & $0.22 \mathrm{n} . \mathrm{s}$ & $0.14 \mathrm{n} . \mathrm{s}$ & $0.25 \mathrm{n} . \mathrm{s}$ & $0.05 \mathrm{n} . \mathrm{s}$ \\
& & & & & & &
\end{tabular}

R.i: R. irregularis; T.v: T. viride; PHE: Phenanthrene. n.s: Not significant. ${ }^{*} \mathrm{P}<0.05 ; * * \mathrm{P}<0.01,{ }^{* * *} \mathrm{P}<0.001$

Table 4. Microbial biomass by whole cell fatty acids analysis (nmol g-1 dry soil).

\begin{tabular}{clcccccccc}
\hline Compartments & Control & R.i & T.v & R.i + T.v & PHE & R.i + PHE & T.v + PHE & R.i + T.v + PHE \\
\hline \multirow{6}{*}{ RHC } & AMF & $0.82 \pm 0.19$ & $26.36 \pm 6.83$ & $1.36 \pm 0.05$ & $8.87 \pm 3.8$ & $1.18 \pm 0.23$ & $5.08 \pm 3.07$ & $0.91 \pm 0.14$ & $1.97 \pm 0.16$ \\
& SF & $5.09 \pm 1.11$ & $9.9 \pm 3.94$ & $7.17 \pm 0.44$ & $5.81 \pm 0.16$ & $13.48 \pm 6.46$ & $14.28 \pm 11.20$ & $13.96 \pm 8.5$ & $6.11 \pm 0.68$ \\
& Gram (-) & $4.51 \pm 1.41$ & $4.82 \pm 0.35$ & $6.43 \pm 0.23$ & $6.03 \pm 0.35$ & $5.56 \pm 0.33$ & $4.48 \pm 1.1$ & $5.14 \pm 0.37$ & $6.82 \pm 0.08$ \\
& Gram (+) & $7.93 \pm 2.07$ & $8.44 \pm 0.3$ & $11.66 \pm 2.27$ & $10.92 \pm 2.27$ & $10.51 \pm 0.16$ & $8.58 \pm 1.73$ & $10.31 \pm 0.49$ & $12.67 \pm 015$ \\
& Actinomycetes & $1.71 \pm 0.43$ & $1.33 \pm 0.22$ & $2.66 \pm 0.13$ & $2.33 \pm 0.15$ & $2.11 \pm 0.29$ & $1.71 \pm 0.53$ & $1.86 \pm 0.22$ & $2.78 \pm 0.06$ \\
\hline \multirow{6}{*}{ AMF } & $0.62 \pm 0.05$ & $17.44 \pm 7.12$ & $0.74 \pm 0.08$ & $12.95 \pm 1.95$ & $0.67 \pm 0.03$ & $10.74 \pm 4.08$ & $0.88 \pm 0.16$ & $0.43 \pm 0.22$ \\
& AF & $1.19 \pm 0.4$ & $1.31 \pm 0.14$ & $1.42 \pm 0.37$ & $2.14 \pm 1.34$ & $1.30 \pm 0.27$ & $1.29 \pm 0.31$ & $1.29 \pm 0.24$ & $1.48 \pm 0.82$ \\
HC & Gram (-) & $3.03 \pm 0.29$ & $3.5 \pm 0.13$ & $3.58 \pm 0.2$ & $4.08 \pm 0.13$ & $2.71 \pm 0.25$ & $3.33 \pm 0.35$ & $3.20 \pm 0.53$ & $2.49 \pm 0.72$ \\
& Gram(+) & $7.76 \pm 0.34$ & $8.25 \pm 0.31$ & $8.51 \pm 2.02$ & $8.06 \pm 0.42$ & $7.96 \pm 0.27$ & $8.34 \pm 0.63$ & $8.09 \pm 0.71$ & $7.70 \pm 0.38$ \\
& Actinomycetes & $1.37 \pm 0.09$ & $1.48 \pm 0.09$ & $1.66 \pm 0.15$ & $1.57 \pm 0.29$ & $1.59 \pm 0.13$ & $1.77 \pm 0.07$ & $1.66 \pm 0.3$ & $0.96 \pm 0.48$ \\
& & & & & & & & & \\
\hline
\end{tabular}

R.i: R. irregularis; T.v: T. viride; PHE: Phenanthrene; RHC: Root and hyphal compartment; HC: Hyphal compartment. AMF: Fatty acid for arbuscular mycorrhizal fungi; S.F: Fatty acid for saprophytic fungi ; Gram (-): Fatty acid for gram negative bacteria; Gram (+): Fatty acid for gram positive bacteria; Actinomycete: Fatty acid for Actynomycetes; . The values shown are the mean \pm standard deviation of triplicates. 

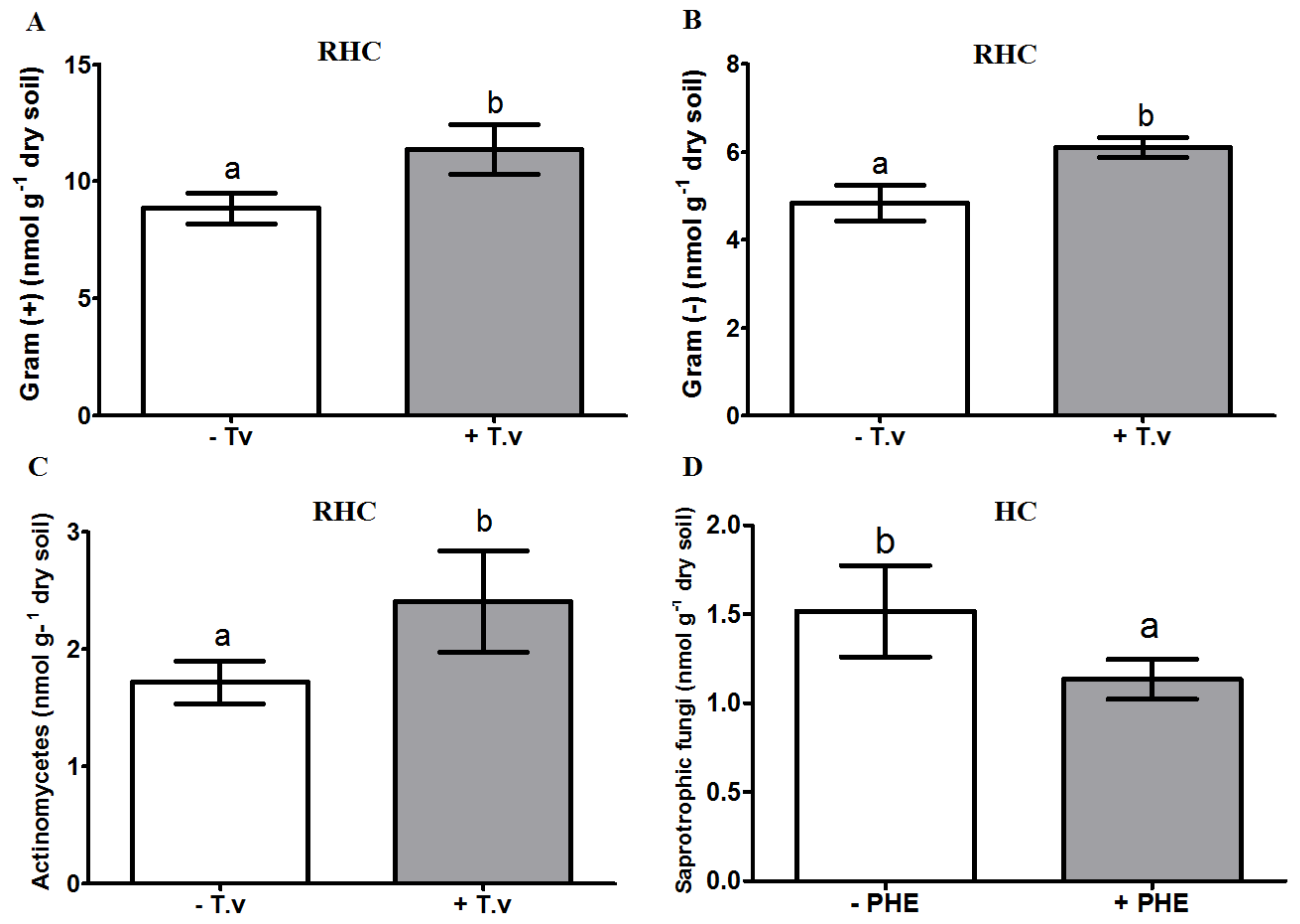

Figure 3. Factor treatments means for fatty acids profile of different organisms in the different compartments. A) Effect of $T$. viride on gram negative fatty acid profile in RHC. B) Effect of $T$. viride on gram positive fatty acid profile in RHC. C) Effect of T. viride on actinomycetes fatty acid profile in RHC. D) Effect of phenanthrene on saprotrophic fungi in HC. RHC: Root and hyphal compartments; HC: Hyphal compartments; T.v: T. viride; PHE: Phenanthrene. Different letters indicate significant factor effects and error bars represent standard error of the mean. 
A

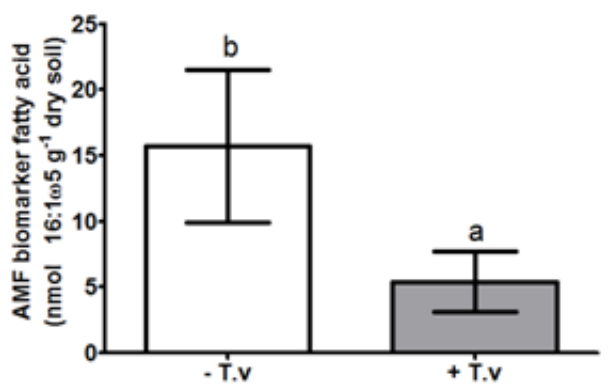

C

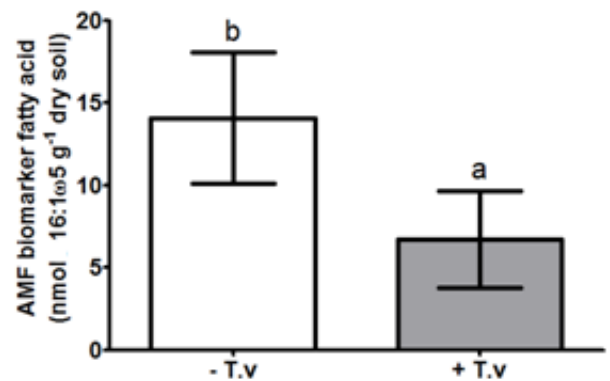

B

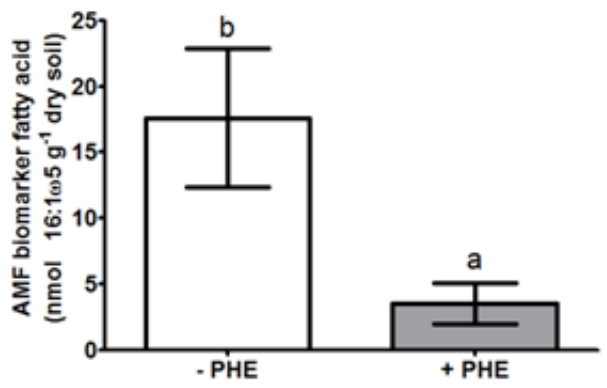

D

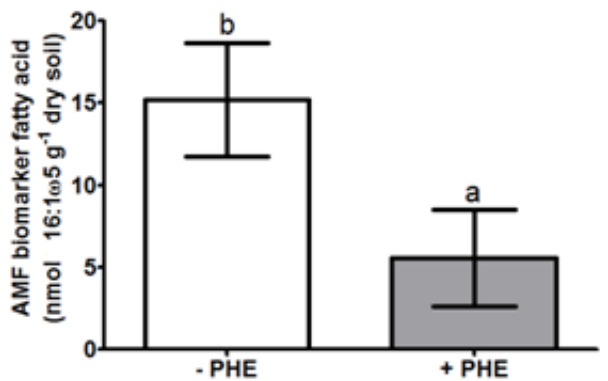

Figure 4. Factor treatments means for 16:105 (AMF fatty acid) in the different compartments. A) Effect of T.viride in RHC. B) Effect of phenanthrene in RHC. C) Effect of T.viride in HC. D) Effect of phenanthrene in HC; RHC: Root and hyphal compartments; HC: Hyphal compartments; T.v: T. viride; PHE: Phenanthrene. Different letters indicate significant factor effects and error bars represent standard error of the mean.

\section{Discussion}

Here we show that soil inoculation with the AM fungus $R$. irregularis and the saprotrophic fungus T. viride did not mitigate Phenanthrene toxicity in maize as expected. On the contrary dual inoculation with $R$. irregularis and $T$. viride resulted in maize plant growth suppression independent of Phenanthrene application. These results reject our main hypothesis that dual inoculation with $R$. irregularis and $T$. viride improve plant growth and mitigate plant stress from Phenanthrene application to a higher extent than from single fungal inoculation.
The observed maize shoot and root growth suppression when grown in Phenanthrene spiked soil is in accordance with other reports on this matter, which has been suggested to be linked with oxidative damage through reactive oxygen species (Salehi-Lisar and Deljoo, 2015). Soil inoculation with AM fungus and Trichoderma spp has been found to mitigate toxic effects of Phenanthrene on plants linked to dissipation in terms of bioaccumulation and/or degradation (Wu et al., 2011). In the present study single or dual inoculation with $R$. irregularis and $T$. viride had no effect on the Phenanthrene toxicity in maize, which may be due to differences in environmental conditions and fungal species employed in these 
studies. On the contrary Phenanthrene application decreased both AM root and soil colonization and $T$. viride population density, which maybe caused from a direct toxic effect. Another explanation is the possible reduction in root exudation after Phenanthrene application as reported by Muratova et al. (2009) who found that $100 \mathrm{mg}$ Phenanthrene $\mathrm{kg}^{-1}$ soil decreased root exudation of carbohydrates, amino acids and carboxylic acids in Sorghum bicolor. Phenanthrene had no effects on the abundance of soil bacteria, which is in contrast to the observed effects on AM fungi and T. viride. Phenanthrene also reduced the abundance of saprotrophic fungi in terms of biomarker fatty acids though only significantly in the HCs. These results suggest a differential response of bacteria and fungi to Phenanthrene, which however needs to be further addressed. The observed maize growth promotion from inoculation with $R$. irregularis is a common mycorrhiza response in maize though depending on maize genotype and phosphorus fertilization (Banerjee et al., 2017; Sawers et al., 2017). In the present study dual inoculation with $R$. irregularis and $T$. viride resulted in plant growth suppression compared to single inoculation with $R$. irregularis. Trichoderma spp. are also known plant growth promoters (Herrera-Jiménez et al., 2018), though in the present study $T$. viride only promoted root growth and not shoot growth. Improved plant growth promotion from dual inoculation with AM fungi and Trichoderma spp has been reported in other plants such as pepper (Bhuvaneswari et al., 2014) and cucumber (Chandanie et al., 2009), but to our best knowledge plant growth suppression from dual inoculation with AM fungi and Trichoderma spp. as observed in the present study has not been reported before.

The observed maize plant growth suppression after dual fungal inoculation coincided with mutual inhibition between $R$. irregularis and $T$. viride independent of Phenanthrene application. Inoculation with $T$. viride especially reduced the growth of $R$. irregularis in the soil, which may impaired the AM hyphal P uptake capacity of $R$. irregularis and consequently resulting in plant growth suppression since the plant benefits from hosting the fungal partner was lower than the costs in terms of photosynthates. The underlying mechanism involved in the inhibition of $R$. irregularis soil colonization by $T$. viride needs to be further addressed, but may be linked to competition for soil nutrients as also suggested by Green et al. (1999). Other possible modes of interactions include mycoparasitism and/or production of antagonistic secondary compounds or changes in soil microbial community. In other studies of AM fungi Trichoderma interactions both inhibition and promotion of AM root colonization from inoculation with different Trichoderma species has been observed (Contreras-Cornejo et al., 2016; Dehariya et $a l, 2015$ ). Our results showed that $R$. irregularis reduced the population density of $T$. viride in the RHC, but had no effect in the HCs, indicating that the effects of $R$. irregularis maybe plant mediate perhaps from reduced root exudation, which is a known plant mycorrhiza response (Graham et al., 1981). However, this needs to be further examined. On the contrary mycelium of $R$. irregularis has been found to reduce the population density of T. harzianum in root-free soil (Green et al., 1999), showing that direct effects of $R$. irregularis on the population density of $T$. harzianum occurred. In the case of soil growth of $R$. irregularis similar inhibition from $T$. viride inoculation was observed independent of the absence or presence of roots, which suggest a direct effect of $T$. viride on $R$. irregularis, which was also the case in the study of Green et al. (1999), who suggested competition for nutrients as the main mode of interaction.

In general, the outcome of AM-Trichoderma interactions seems to be complex depending on fungal species and genotypes as well as the host plant and environmental conditions. When developing fungal consortia for different purposes such as pest biocontrol, 
biofertilization and/or bioremediation it is important to consider this complexity and ensure compatibility between the fungi in question. However, it is also important to consider that mutual inhibition between AM and saprotrophic fungus may also result in plant growth promotion as was shown when dually inoculating the AM $R$. irregularis and the saprotrophic fungus $\mathrm{Clo}$ nostachysrosea associated with tomato grown in soil amended with organic matter (Ravnskov et al., 2006).

\section{Conclusion}

In conclusion our results show that mutual inhibition between the plant growth promoting fungi $R$. irregularis and $T$. viride can result in plant growth suppression emphasizing the importance to test for fungal compatibility when AM fungi and Trichoderma spp. are dually inoculated in order to achieve suitable strategies for PAH bioremediation.

\section{Acknowledgements}

Financial support of this study was provided by the Fondo Nacional de Desarrollo, Científico y Tecnológico, FONDECYT 1130662, and CONICYT Doctoral Fellowship 21120476.

\section{References}

Arriagada, C., Aranda, E., Sampedro, I., GarciaRomera, I., Ocampo, J.A. 2009. Interactions of Trametes versicolor, Coriolopsis rigida and the arbuscular mycorrhizal fungus Glomus deserticola on the copper tolerance of Eucalyptus globulus. Chemosphere. 77, 73-278.

Banerjee, A., Bareh D.A., Joshi, S.R. 2017. Native microorganisms as potent bioinoculants for plant growth promotion in shifting agriculture (Jhum) systems. J. Soil Sci. Plant Nutr. 17, 127-140.
Bhuvaneswari, G., Reetha, S., Sivaranjani, R., Ramakrishnan, K. 2014. Effect of AM fungi and Trichoderma species as stimulations of growth and morphological character of chilli (Capsicum annuum L.). Int. J. Curr. Microbiol. Appl. Sci. 3, 447-455.

Chandanie, W.A., Kubota, M., Hyakumachi, M. 2009. Interactions between the arbuscular mycorrhizal fungus Glomus mosseae and plant growth-promoting fungi and their significance for enhancing plant growth and suppressing damping-off of cucumber (Cucumis sativus L.). Appl. Soil Ecol. 41, 336-341.

Contreras-Cornejo, H.A., Macías-Rodríguez, J., delVal, E., Larsen, J. 2016. Ecological functions of Trichoderma spp. and their secondary metabolites in the rhizosphere: interactions with plants. FEMS Microbiol. Ecol. 92, fiw036.

Cornejo, P., Meier, S., García, S., Ferrol, N., Durán, P., Borie, F., Seguel, A. 2017. Contribution of inoculation with arbuscular mycorrhizal fungi to the bioremediation of a copper contaminated soil using Oenothera picensis. J. Soil Sci. Plant Nutr. $17,14-21$

Dehariya, K., Shukla, A., Ganaie, M.A., Vyas, D. 2015. Individual and interactive role of Trichoderma and Mycorrhizae in controlling wilt disease and growth reduction in Cajanus cajan caused by Fusarium udum. Arch. Phytopathology. Plant Protect. 48, 50-61.

Elad, Y., Chet, I., Henis, Y.A. 1981. Selective medium for improving quantitative isolation of Trichoderma spp. from soil. Phytoparasitica. 9, 59-67.

Frostegård, Å., Bååth, E. 1996. The use of phospholipid fatty acid analysis to estimate bacterial and fungal biomass in soil. Biol. Fertil. Soils. 22, 5965.

Fuentes, A., Almonacid, L., Ocampo, J.A., Arriagada, C. 2016. Synergistic interactions between a sapro- 
phytic fungal consortium and Rhizophagus irregularis alleviate oxidative stress in plants grown in heavy metal contaminated soil. Plant Soil. 407, 355-366.

Gao, Y., Li, Q., Ling, W., Zhu, X. 2011. Arbuscular mycorrhizal phytoremediation of soils contaminated with phenanthrene and pyrene. J. Hazard. Mater.185, 703-709.

Giovanetti, M., Mosse, B. 1980. Evaluation of techniques for measuring vesicular arbuscular mycorrhizal infection in roots. New Phytol. 84, 489-500.

Graham, J.H., Leonard, R.T., Menge, J.A. 1981. Membrane-mediated decrease in root exudation responsible for phorphorus inhibition of vesicular-arbuscular mycorrhiza formation. Plant Physiol. $68,548-552$.

Green, H., Larsen, J., Olsson, P.A., Jensen, D.F., Jakobsen, I. 1999. Suppression of the biocontrol agent Trichoderma harzianum by mycelium of the arbuscular mycorrhizal fungus Glomus intraradices in root-free soil. Appl. Environ. Microbiol. 65, 1428-1434.

Herrera-Jiménez, E., Alarcón, A., Larsen, J., Ferrera-Cerrato, R., Cruz-Izquierdo, S., FerreraRodríguez, M.R. 2018. Comparative effects of two indole-producing Trichoderma strains and two exogenous phytohormones on the growth of Zea mays L., with or without tryptophan. J. Soil Sci. Plant Nutr. 18, 188-201.

Liu, R., Dai, Y., Sun, L. 2015. Effect of Rhizosphere enzymes on phytoremediation in PAH-contaminated soil using five plant species. PLoSOne. 10, 1-14.

Maliszewska-Kordybach, B. 1999. Sources, concentrations, fate and effects of polycyclic aromatic hydrocarbons (PAHs) in the environment. Part A: PAHs in air. Polish J. Environ. Studies. 8, 131136.
Mineki, S., Suzuki, K., Iwata, K., Nakajima, D., Goto, S. 2015. Degradation of polyaromatic hydrocarbons by fungi isolated from soil in Japan. Polycycl. Aromat. Comp. 35, 120-128.

Muratova, A., Golubev, S., Wittenmayer, L., Dmitrieva, T., Bondarenkova, A., Hirche, F., Merbach, W., Turkovskaya, O. 2009. Effect of the polycyclic aromatic hydrocarbon phenanthrene on root exudation of Sorghum bicolor(L.) Moench. Environ. Exp. Bot. 66, 514-521.

Olsson, P.A., Thingstrup, I., Jakobsen, I., Bååth, E. 1999. Estimation of the biomass of arbuscular mycorrhizal fungi in a linseed field. Soil Biol. Biochem. 31, 1879-1887.

Parrish, Z.D., White, J.C., Isleyen, M., Gent, M.P.N., Iannucci-Bergerb, W., Eitzer, B.D., Kelsey, J.W., Incorvia, M. 2006. Accumulation of weathered polycyclic aromatic hydrocarbons (PAHs) by plant and earthworm species. Chemosphere. 64, 609-618.

Phillips, J., Hayman, D. 1970. Improved procedure for clearing roots and staining parasitic and vesiculararbuscular mycorrhizal fungi for rapid assessment of infection. Trans. Br. Mycol. Soc. 55, 158-161.

Ravnskov, S., Jensen, B., Knudsen, I.M., Bødker, L., Jensen, D.F., Karliński, L., Larsen, J. 2006. Soil inoculation with the biocontrol agent Clonostachys rosea and the mycorrhizal fungus Glomus intraradices results in mutual inhibition, plant growth promotion and alteration of soil microbial communities. Soil Biol. Biochem. 38, 3453-3462.

Ratledge, C., Wilkinson, S.G. 1988. Microbial Lipids, Vol. 1. Academic Press, New York, NY.

Salehi-Lisar, S., Deljoo, S. 2015. The physiological effect of fluorene on Triticum aestivum, Medicago sativa, and Helianthus annus. Cogent Food Agric. $1,1020189$. 
Sasser, M. 1990. Identification of bacteria through fatty acid analysis. Methods Phytobacteriol. 199204.

Sawers, R.J., Svane, S.F., Quan, C., Grønlund, M., Wozniak, B., Gebreselassie, M.N., González-Muñoz, E., Chávez Montes, R.A., Baxter, I., Goudet, J., Jakobsen, I., Paszkowski, U. 2017. Phosphorus acquisition efficiency in arbuscular mycorrhizal maize is correlated with the abundance of rootexternal hyphae and the accumulation of transcripts encoding PHT1 phosphate transporters. New Phytol. 214, 632-643.

Smith, S.E., Read, D.J. 2008. Mycorrhizal symbiosis. 3rd edn. Academic Press.

Vácha, R., Čechmánková, J., Skála, J. 2010. Polycyclic aromatic hydrocarbons in soil and selected plants. Plant Soil Environ. 56, 434-443.
Visauta, B. 1998. Análisis estadístico con SPSS para Windows. Estadística multivariante. McGraw Hill, España, 224 pp.

Wu, F.Y., Yu., X.Z., Wu, S.C., Lin, X.G., Wong, M.H 2011. Phenanthrene and pyrene uptake by arbuscular mycorrhizal maize and their dissipation in soil. Journal of Hazardous Materials. 187, 341347.

Yadav, A., Yadav, K., Aggarwal, A. 2015. Impact of arbuscular mycorrhizal fungi with Trichoderma viride and Pseudomonas fluorescens on growth, yield and oil content in Helianthus annuus L. J. Essent. Oil Bear. Plants. 18, 444-454. 